\title{
INTENSIDADE DE PARASITISMO POR EurYtrema SP EM BOVINOS DA MESORREGIÃO DO SUDOESTE PAULISTA*
}

\author{
PARASITE LOAD IN EURYTREMA SP INFECTED CATTLE FROM SOUTHWESTERN \\ LOCALITIES OF THE SĀO PAULO
}

Pacífico António Diniz BELEM'; Mauro Rodrigues de OLIVEIRA²; Carlos Roberto PADOVANP

\begin{abstract}
RESUMO
Após cxames macroscópicos de 485 pâncreas de bovinos procedentes da mesorrcgiðo do Sudocstc Paulista, obtivcram-sc 41 casos dc parasitismo por Eurytrema sp c, a scguir, foram contados os cspécimes albergados. Verificou-se que a intensidade de parasitismo é baixa e que a proporçăo de animais infectados, independentemente da carga parasitária, situa-se cntre 7,2\% c 9,8\%.
\end{abstract}

UNITERMOS: Eurytrema; Carga parasitária; Infecção; Bovinos

\section{INTRODUÇÃO}

O primciro diagnóstico de infecçăo por Eurytrema sp, cm ruminantes, no Brasil, foi feito por HORTA ${ }^{8}$ (1918). Hoje, o parasita e relatado nos Estados de Minas Gerais, Rio de Janciro, São Paulo, Paraná, Santa Catarina, Rio Grande do Sul, Mato Grosso do Sul ${ }^{6}$ c Mato Grosso ${ }^{7,19}$.

No Vale do Paraiba, Eslado de Săo Paulo, a infecçāo por Eurytrema sp cm bovinos é endêmica ${ }^{2.10,11,15,16,18}$ mas, no tocante ao resto do Estado, sabc-sc apenas que: $1^{\circ}$ ) COSTA ct $\mathrm{al}^{4,5}(1978,1979)$ encontraram, à necrópsia, quatro bezcrros parasitados na rcgiðo de Jaboticabal; $2^{\circ}$ ) CORREA ct al. ${ }^{3}$ (1984) diagnosticaram a refcrida parasitose $\mathrm{cm}$ diversas vacas compradas cm Cianortc, Paraná, após tercm sido trazidas para Angatuba, SP; $3^{\circ}$ ) MATTOS JUNIOR c VIANNA" (1987) constataram a prescnça de Eurytrema $\mathrm{cm}$ pâncreas de bovinos abatidos no Valc do Ribcira; $4^{\circ}$ ) OLIVEIRA c MATSUMOTO ${ }^{12}$ (1985), necropsiando 74 animais mestiços holandês-zcbu, na faixa ctária de 8 a 20 meses, durante o periodo de dezcmbro de 1979 a dezembro de 1983, reportaram o achado de $14,86 \%$ de prevalência c 146,28 de intensidade média de infeç̧ão; $5^{\circ}$ ) SANTOS ${ }^{17}$ (1986) identificou $42,6 \%$ de parasitismo $\mathrm{cm}$ um lote de 129 bovinos abatidos $\mathrm{cm}$ Barretos, lodos oriundos de uma mesma fayenda da Cidade de Sæo Manocl.

Tendo $\mathrm{cm}$ vista a alta prevalência verificada $\mathrm{cm}$ bovinos de Săo Manocl, decidiu-sc cfetuar um cstudo mais aprofundado sobre a intensidade de parasitismo $\mathrm{cm}$ animais da mesorregião do Sudoeste Paulista a fim de se avaliar melhor a importância da infeç̧̋̄o em estudo. Os resultados desta investigaça achamsc aqui consignados.

\section{MATERIAL E MÉTODO}

O matcrial desta pesquisa foi obtido de 483 bovinos de ambos os sexos, pertencentes a diferentes raças c oriundos de diferentes propricdades rurais, que foram abatidos cm $S x_{0}$ Manocl, Estado de São Paulo, cntrc 23/1 1/87 a 25/1/88, além de outros dois nccropsiados na Faculdade de Mcdicina Veterinária c Zootccnia da UNESP/Botucatu (FMVZ). Todos os animais cram adultos, criados $\mathrm{cm}$ regime de pasto $\mathrm{c}$ procedentes de municípios da mesorregiăo do Sudoestc Paulista.

Após a cvisccraçæo dos animais, os pâncreas cram cxamina. dos c, quando parasitados, acondicionados $\mathrm{cm}$ sacos plásticos individuais previamente identificados a fim de serem transporLados para a FMVZ, onde sc procedia à contagem dos espécimes albergados. Para isto, cada um era colocado $\mathrm{cm}$ uma bandeja individual, libertado das partes restantes de vísccras adjaccntcs, lavado, c toda a água usada cra passada através de penciras de nylon para scparação dos trematóides desgarrados. A scguir, os canais pancréticos cram abertos com auxilio de uma tcsoura e os helmintos retirados e contados.

De posse dos dados relativos à intensidade de parasitismo, inicialmente foram feitas comparaçðes entre cles c outros registrados na litcratura. A seguir, os valores das cargas parasitárias cncontradas foram reunidos àqueles dos animais não parasitados ( $n^{e}$ de parasitos $=0$ ) e construído o intcrvalo de confiança para a proporçao de parasitados a $95 \%$ de confiabilidadc. Por fim, considerando-se apenas os valores dos animais parasitados, testou-sc, por meio do teste de Kolmogorov e Smimov para uma amostra, sc cles poderiam ser cstudados por um modclo de distribuiçăo gcométrica iruncada ${ }^{13}$.

\section{RESULTADOS E DISCUSSĀO}

O númcro de espécimes de Eurytrema sp por bovino e informaçð̌es referentes aos abates que permitiram a obtençāo do matcrial para os fins deste estudo, sāo apresentados na Tab 1. Os dois animais nocropsiados procederam de Cerqueira César (SP) c Sāo Manocl c, respectivamente, albergavam nos pâncreas 42 c 31 trematóides.

Para se ter uma idéia da magnitude do grau de infecçāo por Eurytrema sp, nos animais desta pesquisa, é neccssário compará-lo àqucles descritos na literatura 1,2, 12,14, 18. Todavia,

\footnotetext{
- Parte da tesc: BELf́M, P.A.D. Aspectos ligados ao dlagnóstico da infecço por Eurytrema sp em bovinos. Botucalu, 1991. Tese (Doutorado) - Faculdade de Medicina Veterinária e Zootecnia, Universidade Estadual Paulista - Campus de Boucalu.

1-Professor Adjunto - Universidade Federal de Viçosa

2-Professor Adjunto - Fraculdade de Medicina Veterinária c Zootecnia da UNESP, Campus de Botucatu

3-Professor Adjunto - Instituto de Biocéncia da UNESP, Campus de Bolucatu
} 
BELÉM, P.A.D.; OLIVEIRA, M.R.: PADOVANI, C.R. Intensidade de parasitismo por Eurytrema sp em bovinos da mesorregiāo do Sudoesıc Paulista. Braz.

J. vet. Res. anim. Scl., São Paulo, v.29, p. 366-368, 1992 Suplemento.

por se tratar de estudo conduzido $\mathrm{cm}$ bovinos adultos $\mathrm{e}$ de matadouro, os resultados obtidos nāo poderiam ser comparados aos de todas as publicaçð̄es acima citadas porque as duas últimas encerram informaçocs sobre a intensidade de parasitismo $\mathrm{cm}$ animais jovens apenas. Ademais, tal como se constata pelo trabalho de KONO e FUKUYOSHI' (1966), bovinos com idade inferior a um ano apresentam-se menos frequentemente parasitados por Eurytrema sp e, pode-se supor, devem também exibir menorcs cargas parasitárias, haja visto os baixos valores descritos por VIANNA ${ }^{18}$ (1985) c OLIVEIRA; MATSUMOTO ${ }^{12}$ (1985). Deste modo, nāo parece corrcto estabelecer paralclos entre intensidade de parasitismo de animais jovens e adultos, sobretudo se considerarmos que os primeiros, lactantes durante boa parte de vida, acabam se cxpondo menos à infecçðo cm apreço.

Confrontando-sc, pois, o número médio de parasitas encontrado com os descritos por PURVIS ${ }^{14}$ (1933); BASCH $^{1}$ (1965) e CAMPOS ct al. ${ }^{2}$ (1974), constata-sc que cle se mostra aparentemente bem inferior $\mathrm{cm}$ todos os casos. No que tange a CAMPOS et al. ${ }^{2}$ (1974), o teste de comparaçāo de médias conduz à confirmação da maior magnitude da intensidade média de parasitismo a favor do autor $\mathrm{cm}$ questăo, cmbora esta comparaçăo possa também ser vista com reservas porquanto CAMPOS et al. ${ }^{2}$ (1974) não mencionaram a época durante a qual colhcram o matcrial. Todavia, é possivel também que tal diferença possa ser atribuída a características adversas da mesorregiāo do Sudocste Paulista que acarretem a reduçāo da população de hospedciro intermediário c, por conscguinte, dificultem no fechamento do ciclo biológico do Eurytrema sp.

Em consonância com o que acaba de ser discutido, ao sc tomar os valores das cargas parasitárias de todos os animais (infectados ou nåo) verifica-se que a probabilidade de sc encontrar casos da referida infecçăo (p) corresponde ao intervalo de confiança

TABELA 1

Número de bovinos abatidos, procedência, freqüência observada de casus de infecção natural por Eury trema sp e respectivos valores das cargas parasitárias verificadas entre 23/1 1/87 e 25/1/88 na cidade de São Manocl, São Paulo.

\begin{tabular}{|c|c|c|c|c|}
\hline \multirow[b]{2}{*}{ Data } & \multirow{2}{*}{$\begin{array}{l}\text { N de bo- } \\
\text { vinos } \\
\text { abatidos }\end{array}$} & \multirow[b]{2}{*}{ Procedência } & \multicolumn{2}{|c|}{ Casos de curitrematose } \\
\hline & & & $\begin{array}{l}\text { Iircq. } \\
\text { observ. }\end{array}$ & $\begin{array}{l}\text { Cargas parasi- } \\
\text { tárias obscrv. }\end{array}$ \\
\hline $23 / 11 / 87$ & 60 & Säo Manocl & 6 & $\begin{array}{l}143,143,218 \\
254,391 \text { } 477\end{array}$ \\
\hline $07 / 12 / 87$ & 70 & Pardinho & 9 & $\begin{array}{r}34,44,46 \\
72,124,132 \\
148,210 \text { e } 231\end{array}$ \\
\hline $04 / 01 / 88$ & $\begin{array}{l}69 \\
21\end{array}$ & $\begin{array}{l}\text { Agudos } \\
\text { Itapeva }\end{array}$ & - & - \\
\hline $050 / 1 / 88$ & 68 & Itspeva & 3 & 4,35 e 712 \\
\hline $11 / 01 / 88$ & 67 & Itapeva & 1 & 1165 \\
\hline $13 / 01 / 88$ & 47 & $\begin{array}{l}\text { Itapeva c } \\
\text { Itaí }\end{array}$ & 1 & 315 \\
\hline $14 / 01 / 88$ & 67 & $\begin{array}{l}\text { Itapeva e } \\
\text { ILaí }\end{array}$ & 5 & $\begin{array}{r}203,356,357 \\
578 \text { c } 1159\end{array}$ \\
\hline $25 / 01 / 88$ & 14 & Timburi & 14 & $\begin{array}{r}167,177,204 \\
228,323,342 \\
372,394,580 \\
662,759,943 \\
1045 \mathrm{e} 1280\end{array}$ \\
\hline Total & 483 & - & 39 & - \\
\hline
\end{tabular}

$0,072 \leq \mathrm{P} \leq 0,098$. Finalmentc, no caso dos animais parasitados, a lei de probabilidade de ocorrência do número de cspécimes dc Eurytrema sp por bovino (NP), ajustada às obscrvaçð̄cs $(\mathrm{D}=0,1864, P>0,10)$ c definida pela cquação:

$P(N P=x)=0,0027176.0,9972824^{x \cdot 1}$ para $x=1,2,3, \ldots$ cvidencia quc, na regiåo $\mathrm{cm}$ estudo, cargas parasitárias baixas têm maior probabilidade de serem detectadas que as altas (Fig. I).

\section{CONCLUSÃO}

1') a intensidade média de parasitismo é baixa, cm comparaçāo com outras descritas na literatura;

$\left.2^{2}\right)$ estima-sc que a proporçāo de animais infectados, independentemente da carga parasitária, situa-se entre 7,2\% c 9,8\%;

3') a distribuição de probabilidades de ocorrência da variável número de cspécimes de Eurytrema sp por bovino (NP) obcdece à distribuição gcométrica truncada definida por:

$P(N P=x)=0,00027176.0,99722824^{*-1}$, scndo $x=1,2,3, \ldots$;

$\left.4^{*}\right)$ cargas parasitárias baixas predominam sobre as altas $c$ têm maior probabilidade de serem encontradas.

\section{SUMMARY}

By macroscopic examination, 485 pancreas of calle from Southwestern localities of Sāo Paulo State were checked in order to count the Furytrema sp specimens. It was concluded that the average parasite load is low in the region under study and the occurence ranged between $7.2 \%$ and $9.8 \%$.

UNITER.MS: Furytrema sp; Parasite load; Infection; Cattlc

\section{REFERÊNCIAS BIBLIOGRÁFICAS}

01-BASCH, P. F. Completion of the life cucle of Eurytrema pacreaticum (STRENATIDA: DICROCOELIIDAE). J. Parasit., v.51, p.350-5, 1965.

02-CAMPOS, M. S.; RAGUSA, A. L.; MIGUEL, O.; ISHLZUKA, M. M. Correlação entre número de parasitos $\mathrm{c}$ peso de pâncreas $\mathrm{cm}$ bovinos naturalmente infestados por Eurytrema pacreaticum, Janson, 1889 (TREMATODA-DICROCOELIIDAE), diferença de peso entre pâncreas parasitados c não parasitados. Rev. Fac. Med. Vet. Zoot. Univ. S. Paulo, v.11, p. 295-9. 1974.

03-CORREA, W. M.; CORREA.C. N. M.; FERREIRA, A. C.; PAES, A. C. Eurytrema pacreaticum: clínica c diagnóstico $\mathrm{cm}$ bovinos. Hora Vet., v.4, n.19. p.31-4, 1984.

04-COSTA, A. J.; CAMPOS, M. S.; KASAI, N.; PAULILLO, A. C.; COSTA, A.F. Estudo preliminar sobre helmintoses gastrointestinais de bovinos no município de Jaboticabal, Estado de São Paulo, Brasil. Cientínca, v.6, p.443-9, 1978.

05-COSTA, A. J. MACHADO, R. Z.; KASAI, N. lista de helmintos parasitos de animais domésticos da sub-regiäo de Jaboticabal, Estado de São Paulo. Clentífica, v.7, p. 281-6, 1979.

06-COSTA, H. M. A.; LEITE, A. C. R.; GUIMARĀES, M. P.; LIMA, W.S. Distribuição de helmintos parasitos de animais domésticos no Brasil. Arq. bras. Med. Vet. Zootec., v.38, p. 465-79, 1986.

07-FERNANDES, B.F.; BUSETTI, E.T. Algumas espécies de parasitas novas para a fauna paranaensc. Arq. Biol. Tecnol., v.17, p.856, 1974. 
08-HORTA.P.P. Distomatose pancreática c glicosúria cm bovinos. A Levoura, v.22, p.157-8, 1918.

09-KONO, I.; FUKUYOSHI, S. Studies on the existence of eggs of pancreas fluke in the wall of the pancreatic duct and its significance. Bull Fac. Agrc. Kagoshima Univ, v.12, p. 197-206, 1966.

10-MATTOS JUNIOR, D. G. Eurytrema coelomaticum (Glard et Bllkt, 1892) Loos, 1907: alguns aspectos do seu relacionamento com os hospedeiros intermediários e definitivo sob condiçōes experimentais. Itaguaí, 1987. Dissertação (Mestrado) - Instituto de Biologia, Universidade Fodcral Rural do Rio de Janciro.

11-MATTOS JUNIOR, D.G.; VIANNA, S.S.S. O Eurytrema coelomaticum (TREMATODA:DICROCOELIIDAE) no Brasil. Arq. flumin. med. vet., v.2, p. 3-7, 1987.

12-OLIVEIRA, G.P.; MATSUMOTO,T.Prevalência c intensidade de infocçăo por helmintos $\mathrm{cm}$ bovinos da bacia lcitcira da São Carlos, Såo Paulo. Pesq. Agropec. bras., v.20, p. 1415-8, 1985.

13-OSTLE, B. Estatístlca apllcada. 3.ed. Mexico, Limusa - Wiley, 1973.

14-PUR VIS, G. B. On the apparent non pathogenicity of the trematodes Eurytrema pancreaticum (Janson, 1889) and Platynosomum conclnum (Braun, 1901). Vet. Rec.,v.13, p.1063-4, 1933.

15-RAGUSA, A. L.; CAMPOS, M. S. Encontro de provável hospodciro intermediário do F. pancreaticum Janson (1889)
(TREMATODA DICROCOELIIDAE) parasita de bovinos. Atual. vet., v.2, n.50, p.57, 1972.

16-RAGUSA, A. L.; CAMPOS, M. S. Identificação de hospedciro intermediário de Eurytrema coelomaticum (Giard \& Billct, 1889) Loos, 1907, (TREMATODA - DICROCOELIIDAE) $\mathrm{cm}$ fazendas de criaçăo de bovinos, no estado de São Paulo - Brasil. Rev. Fac. Med. Vet. Zoot. Univ. S. Paulo, v.13 p. 269.87, 1936.

17.SANTOS, I. F. Inspeção "post-monem" de bovinos. Exame de pânctcas para a delocçãa de Eurytrema pancreaticum. JANSON, 1889. Ars Vet., v.2, p.107-1, 1986.

18-VIANNA, S. S. S. Técnica coprocópica de sedimentaçāo para concentraçāo de ovos de Eurytrema sp LOOSS, 1907. Săo Paulo, 1985. Tese(Doutorado)-Instituto de Ciências Biológicas, Universidade de Såo Paulo.

19-YAMAMURA, M.H.;ZANON JUNIOR, L.C.; MARQUES, F.C.; VIDOTTO, O. Prevalência de Eurytrema coelomatlcum (GIARD \& BILLET, 1892) Looss, 1907. Trematódco DICROCOELIIDAE, cm bubalinos abatidos $\mathrm{cm}$ Londrina, PR. In: CONGRESSO BRASILEIRO DE MEDICINA VETERINÁRIA, 18., Camboriu, 1982. Anals. Camboriu, SBMV, 1982. P.209.

Reccbido para publicação $\mathrm{cm} 26 / 6 / 92$ Aprovado para publicaçảo $\mathrm{cm} 10 / 9 / 92$

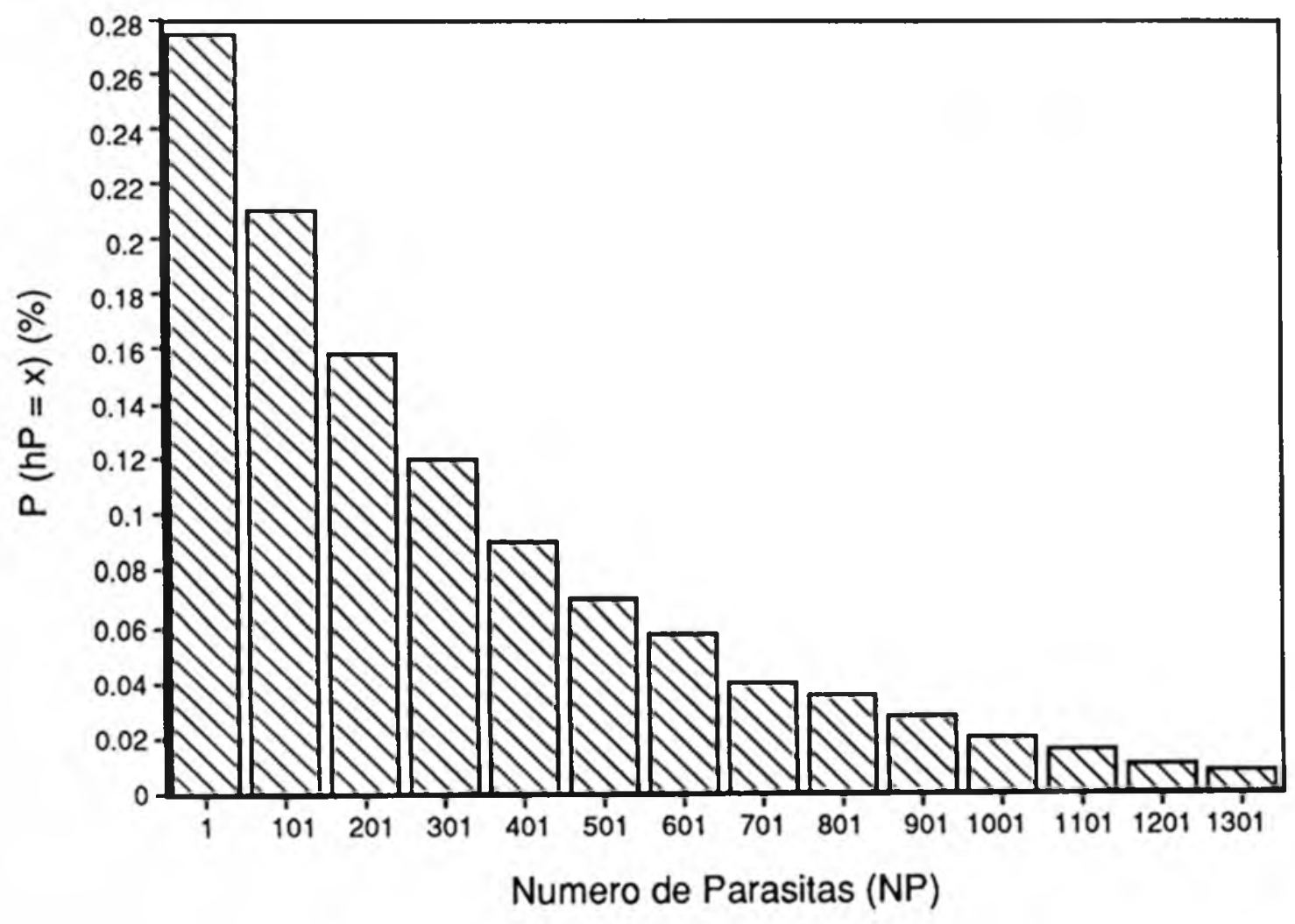

Figun!

Probabilidades associadas (PINP $=\mathrm{xl}$ ) aos valores do número de parasitos (NP) de bovinos infectados naturalmente por Furytrema sp coriundos de municípios da mesorregiazo do Sudocste Paulista. 